\title{
Title
}

\section{Synthesis, structure and electric properties of a new lead-free ferroelectric solid} solution of $(1-x) \mathrm{BaTiO}_{3}-x \mathrm{Bi}\left(\mathrm{Zn}_{2 / 3} \mathrm{Nb}_{1 / 3}\right) \mathrm{O}_{3}$

\section{Authors}

Alisa Paterson ${ }^{\mathrm{a}}$, Hoi Ting Wong ${ }^{\mathrm{a}}$, Zenghui Liu ${ }^{\mathrm{b}}$ Wei Ren ${ }^{\mathrm{b}}$, and Zuo-Guang Ye $\mathrm{e}^{\mathrm{a}, \mathrm{b}}$

\section{Affiliations}

${ }^{\mathrm{a}}$ Department of Chemistry and 4D LABS, Simon Fraser University, 8888 University

Drive,Burnaby, BC, V5A 1S6, Canada

${ }^{\mathrm{b}}$ Electronic Materials Research Laboratory, Key Laboratory of the Ministry of Education \& International Centre for Dielectric Research, Xi'an Jiaotong University, Xi'an, 710049, China

*Corresponding Author. Email Address: zye@sfu.ca ; Tel: 1-778-782-8064; Fax: 1-778782-3765.

\begin{abstract}
Ceramics of a novel lead-free ferroelectric solid solution, (1-x)BaTiO ${ }^{-}$ $x \mathrm{Bi}\left(\mathrm{Zn}_{2 / 3} \mathrm{Nb}_{1 / 3}\right) \mathrm{O}_{3}(\mathrm{BT}-\mathrm{BZN})$, have been prepared with $x=0-0.125$ and their structure and dielectric properties investigated. The effects of BZN substitution for BT on the crystal structure and dielectric and ferroelectric properties are examined. The structure at room temperature is refined to be tetragonal for $x \leq 0.025$, a mixture of rhombohedral and tetragonal phases for $x=0.05$, and pseudo-cubic for $x \geq 0.075$. As the BZN content increases, the Curie temperature $T_{\mathrm{C}}$ of the solid solution decreases, and the phase transition becomes more diffuse. At $x=0.075$, relaxor-like behaviour appears. The diffuseness of the phase transition and the crossover from ferroelectric to relaxor character are attributed to
\end{abstract}


increased cationic disorder arising from the aliovalent coupled substitutions of $\mathrm{Bi}^{3+}$ for $\mathrm{Ba}^{2+}$ and $\left(\mathrm{Zn}_{2 / 3} \mathrm{Nb}_{1 / 3}\right)^{3+}$ for $\mathrm{Ti}^{4+}$. A partial phase diagram of the solid solution is established.

\section{Keywords}

C. Dielectric properties, Ferroelectric properties, D. Perovskites, Relaxor

\section{Introduction}

Ceramics and single crystals of solid solutions with lead titanate, $\mathrm{PbTiO}_{3}(\mathrm{PT})$, as an end member, such as $(1-x) \mathrm{Pb}\left(\mathrm{Mg}_{1 / 3} \mathrm{Nb}_{2 / 3}\right) \mathrm{O}_{3}-x \mathrm{PbTiO}_{3}$ (PMN-PT), exhibit the highest piezoelectric properties, making them excellent materials for high-performance electromechanical sensor and actuator applications. The highest properties are observed at the morphotropic phase boundary (MPB) compositions. These MPB-based materials are compositionally engineered to enhance performance, but this generally results in a decreased Curie temperature $\left(T_{\mathrm{C}}\right)$ [1]. Therefore, MPB systems often possess a decreased temperature range of operation, more temperature-dependent properties, and less polarization stability due to their relatively low $T_{\mathrm{C}}$ and even lower MPB temperature, $T_{\mathrm{MPB}}$ [2]. There have been many efforts to investigate potential high-performance, high $T_{\mathrm{C}}$ materials in order to broaden the temperature range of applications [3-5].

Many lead-based perovskite materials exhibit exceptional piezoelectric and ferroelectric properties; however, there are concerns about lead toxicity, which has led to an increase in the studies of lead-free materials in the past decade. As a result, there has been a particular renewed interest in barium titanate, $\mathrm{BaTiO}_{3}(\mathrm{BT})$, a well-known ferroelectric material, and systems containing BT due to its lead-free nature. The intent of this work is to explore a novel solid solution with BT as one end-member, with the goal of developing a new lead-free ferroelectric material with a $T_{\mathrm{C}}$ greater than that of pure BT. 
There is great interest in bismuth-based perovskites as a substitute for lead, as both $\mathrm{Pb}^{2+}$ and $\mathrm{Bi}^{3+}$ as A-site cations possess a $6 \mathrm{~s}^{2}$ stereochemically active lone pair, which could enhance spontaneous polarization and increase $T_{\mathrm{C}}$. The ionic size of $\mathrm{Bi}^{3+}(1.36 \AA)$ compared to $\mathrm{Pb}^{2+}(1.49 \AA$ ) allows for different A-O bonding motifs, as its smaller size may allow for increased A-site cationic displacement, increasing spontaneous polarization [5]. For instance, $\mathrm{Bi}\left(\mathrm{Zn}_{1 / 2} \mathrm{Ti}_{1 / 2}\right) \mathrm{O}_{3}$ (BZT) has a calculated ionic polarization of over 150 $\mu \mathrm{C} / \mathrm{cm}^{2}$. A more pronounced influence of the $6 \mathrm{~s}^{2}$ lone pair is seen in the A-site bismuth environment in BZT, which is more distorted than that of lead in $\mathrm{PbTiO}_{3}[6]$. However, Bibased perovskites are less stable than $\mathrm{Pb}$-based perovskites and often require high pressure synthetic techniques. Consequently, a number of studies explore Bi-based perovskite compounds by stabilizing them as an end-member in solid solutions with PT [3,4,7].

An enhanced tetragonality $(c / a=1.08)$ and a $T_{\mathrm{C}}$ greater than that of PT have been reported in the solid solution of $(1-x) \mathrm{PbTiO}_{3}-x \mathrm{Bi}\left(\mathrm{Zn}_{2 / 3} \mathrm{Nb}_{1 / 3}\right) \mathrm{O}_{3}(\mathrm{PT}-\mathrm{BZN})$ [8]. In this work, we stabilize the Bi-based perovskite $\mathrm{Bi}\left(\mathrm{Zn}_{2 / 3} \mathrm{Nb}_{1 / 3}\right) \mathrm{O}_{3}$ in a solid solution with $\mathrm{BT}$, to form the lead-free analog to PT-BZN. We have synthesized the novel solid solution (1$x) \mathrm{BaTiO}_{3}-x \mathrm{Bi}\left(\mathrm{Zn}_{2 / 3} \mathrm{Nb}_{1 / 3}\right) \mathrm{O}_{3}$ (BT-BZN) and investigated its structure and dielectric properties.

\section{Materials Preparation and Characterization Methods}

\subsection{Synthesis}

The ceramics of the $(1-x) \mathrm{BaTiO}_{3}-x \mathrm{Bi}\left(\mathrm{Zn}_{2 / 3} \mathrm{Nb}_{1 / 3}\right) \mathrm{O}_{3}[(1-x) \mathrm{BT}-x \mathrm{BZN}]$ solid solution with compositions of $x=0.0$ to 0.15 were prepared by solid state reaction from the commercially available constituent oxides and carbonates. A stoichiometric amount of the starting compounds $\mathrm{BaCO}_{3}\left(99.95 \%\right.$, Alfa Aesar), $\mathrm{TiO}_{2}$ (99.5\%, Alfa Aesar), $\mathrm{Bi}_{2} \mathrm{O}_{3}$ 
(99.9\%, Alfa Aesar), $\mathrm{ZnO}$ (99.9\%, Kojundo Chemicals) and $\mathrm{Nb}_{2} \mathrm{O}_{5}$ (99.9\%, Strem Chemicals), were mixed together, and hand ground with a mortar and pestle in acetone for 1 hour. The mixtures were pressed uniaxially into a pellet and calcined between 1125 and $1350{ }^{\circ} \mathrm{C}$ for 4 to 6 hours to form the desired perovskite phase as verified by $\mathrm{x}$-ray diffraction (XRD). The pellets were then pulverized, hand ground in acetone with a mortar and pestle for 1 hour, and pressed uniaxially into $10 \mathrm{~mm}$ diameter discs for sintering. A bed of sacrificial $(1-x) \mathrm{BT}-x \mathrm{BZN}$ powder of the same composition was used during the sintering process to prevent $\mathrm{Bi}_{2} \mathrm{O}_{3}$ evaporation. The pellets were sintered at temperatures between 1300 to $1375^{\circ} \mathrm{C}$ for 6 hours to form dense ceramics.

\subsection{Structural Analysis}

The sintered ceramics were polished to provide flat, parallel surfaces. XRD was performed on the ceramics using $\mathrm{Cu} \mathrm{K}_{\alpha}$ radiation (Bruker D8 Advance Diffractometer, 40 $\mathrm{mA}, 40 \mathrm{kV}, 0.01^{\circ}$ steps, $2 \theta=15-80^{\circ}$ ). The resulting diffraction data were analyzed using TOPAS Academic Software to perform the Rietveld refinements to determine the symmetry and refine the lattice parameters of the solid solution.

\subsection{Dielectric Measurements}

The two surfaces of the polished ceramics were painted with a layer of silver paste and gold wires were attached to both surfaces of the samples. The dielectric properties of the BT-BZN ceramics were measured as a function of frequency using a Novocontrol Alpha high resolution broadband dielectric analyzer.

\subsection{Ferroelectric and Piezoelectric Measurements}

The polarization - electric field hysteresis loops were displayed at room temperature using a Radiant RT-66 standardized ferroelectric testing system. For 
piezoelectric measurements, the samples were poled at room temperature in silicon oil using a Stanton PS350 high voltage power source. The piezoelectric constants were measured using a Model ZJ-6B quasistatic piezoelectric meter.

\section{Results and Discussion}

\subsection{Structural Analysis}

Figure 1 shows the XRD patterns for the ceramics of $x=0,0.025,0.05,0.075,0.10$, 0.125 , and 0.15 sintered at different temperatures. The XRD patterns indicate that the BTBZN solid solution crystallizes in a pure complex perovskite phase up to a solubility limit of $x=0.125$. An impurity phase appeared when $x \geq 0.15$, as marked with the stars in Figure 1.

The Rietveld refinements were performed on the XRD patterns using the software TOPAS-Academic. The fitting was carried out based on a model of the tetragonal P4mm phase. The refinement fitting results for the $x=0.025$ ceramic are shown in Figure 2. Slight splitting can be seen in the (200) peaks, shown in the inset, confirming the tetragonal symmetry. The calculated lattice parameters of the tetragonal phase and the tetragonality for the $x=0$ and 0.025 ceramics are listed in Table 1 . It can be seen that, with the substitution of BZN for BT, the lattice parameter $a$ increases while $c$ decreases, leading to a decreased tetragonality, which is contrary to what was expected for the effect of BZN substitution.

The XRD pattern for the $x=0.05$ solid solution (Figure 3) shows splitting of the (111) peak and a shoulder on the (200) peak. The splitting of the (111) peak is characteristic of a rhombohedral symmetry while the shoulder on the (200) peak can be 
accounted for by a tetragonal phase. Therefore, the Rietveld refinements for the $x=0.05$ composition are carried out based on a model of coexisting rhombohedral $R 3 c$ and tetragonal P4mm phases. The inset in Figure 3 is an enlarged view to clearly show the characteristic rhombohedral and tetragonal phase splitting, respectively. The tetragonal phase displays a higher intensity than that of the rhombohedral phase, suggesting that the tetragonal phase is in majority.

Due to the absence of peak splitting, the XRD patterns for the ceramics of compositions with $x \geq 0.075$ were fit to a pseudo-cubic symmetry Pm-3m. The calculated lattice parameters of the pseudo-cubic phase for the $x=0.075,0.10$ and 0.125 ceramics are listed in Table 2.

Overall, the XRD structural refinements indicate that increasing BZN in the (1$x$ )BT- $x$ BZN solid solution increases the symmetry of the crystal structure and reduces the tetragonality, despite the population of the B-site of the perovskite by ferroelectrically active cations and the $\mathrm{A}$-site by the $\mathrm{Bi}^{3+}$ ion with a lone electron pair. This is contrary to the results we have seen in the $(1-x) \mathrm{PbTiO}_{3}-x \mathrm{Bi}\left(\mathrm{Zn}_{2 / 3} \mathrm{Nb}_{1 / 3}\right) \mathrm{O}_{3}$ solid solution [8], where the increase in the BZN content results in an increase in structural asymmetry and an increase in the tetragonality. It is possible that the partial substitution of BZN for BT creates a higher degree of chemical disorder (as indicated below by the diffuse and dispersive dielectric properties) which could cancel the normally expected effect of BZN on the enhancement of structural distortion.

\subsection{Dielectric Measurements}

Figure 4 (a-d) show the dielectric constant $\varepsilon^{\prime}$ as a function of temperature for four different compositions of the $(1-x) \mathrm{BT}-x \mathrm{BZN}$ solid solution measured at different 
frequencies. The peak in the temperature dependence of the dielectric constant in Figure 4 (a) and (b) corresponds to the Curie temperature $T_{\mathrm{C}}$ at which the material undergoes a phase transition from the paraelectric, nonpolar phase, to the ferroelectric, polar phase upon cooling. The dielectric measurement results indicate that the $x=0.025$ and $x=0.05$ solid solutions have the $T_{\mathrm{C}}=98 \pm 3^{\circ} \mathrm{C}$ and $T_{\mathrm{C}}=39 \pm 3^{\circ} \mathrm{C}$, respectively. The relatively broad peaks indicate a second-order phase transition, which were confirmed by the measurements performed upon heating that yielded the same $T_{\mathrm{C}}$ as that measured upon cooling, i.e. no thermal hysteresis. Note that the values of $T_{\mathrm{C}}$ (the peak temperatures) do not depend on frequency, indicating a normal ferroelectric phase transition.

In the ceramics of compositions with $x \geq 0.075$, the temperature of maximum permittivity, $T_{\max }$, increases with increasing measurement frequency and the value of $\varepsilon^{\prime}{ }_{\max }$ decreases, suggesting relaxor-like behaviour. It is known that no changes in the crystal structure are observed around $T_{\max }$ in typical relaxor ferroelectrics, so the maxima in permittivity are not attributed to ferroelectric phase transitions [9]. The XRD data of these compositions are consistent with this behaviour, as they fit well to a pseudo-cubic phase model.

In this BT-BZN solid solution system, increasing the BZN content first lowers the $T_{\mathrm{C}}$ below that of pure $\mathrm{BaTiO}_{3}\left(T_{\mathrm{C}}=120^{\circ} \mathrm{C}\right)$. This decrease in $T_{\mathrm{C}}$ is in agreement with the structural changes refined from the XRD results, which indicate a decrease in the tetragonality and an increase in the crystal symmetry at room temperature, as one would expect a structure that is closer to cubic symmetry to possess a lower $T_{\mathrm{C}}$. As the BZN content increases, the maximum of permittivity becomes more diffuse, and eventually begins to exhibit relaxor-like behaviour through its frequency dependent dispersion of the 
dielectric peaks. Therefore, we have found a crossover from the ferroelectric phase to a relaxor state in the BT-BZN solid solution with increasing BZN concentration.

Based on the absence of thermal hysteresis in the dielectric measurements performed upon cooling and heating, the ferroelectric-paraelectric phase transition in the BT-BZN compounds of low BZN concentrations $(x=0.025$ and 0.05$)$ has been classified as second-order, which is different from the phase transition observed in pure BT which is of first-order. The appearance of the second-order transition could be attributed to the chemical disorder introduced by the coupled substitutions of heterovalent cations, i.e. $\mathrm{Zn}^{2+}$ and $\mathrm{Nb}^{5+}$ for $\mathrm{Ti}^{4+}$ on the $\mathrm{B}$-site and $\mathrm{Bi}^{3+}$ for $\mathrm{Ba}^{2+}$ on the $\mathrm{A}$-site of the perovskite structure. This increase in disorder may be responsible for the observed broad second-order phase transition, increased diffuseness in the dielectric properties and the crossover from ferroelectric to relaxor-like behaviour [10]. This change in the ferroelectric phase transition behaviour has been observed in several other $\mathrm{BaTiO}_{3}$-based solid solution systems, such as $\mathrm{Ba}\left(\mathrm{Ti}_{1-x} \mathrm{Sn}_{x}\right) \mathrm{O}_{3}$ [11-16]. Ogihara et al. suggested the relaxor-like behaviour of $\mathrm{BaTiO}_{3^{-}}$ $\mathrm{Bi}\left(\mathrm{Me} \mathrm{Me}^{\prime}\right) \mathrm{O}_{3}$ systems could be a result of weak coupling between nanopolar regions, making it difficult to obtain long-range polar order [14].

The difference in $T_{\max }$ at different frequencies, $\Delta T_{\max }=\left[T_{\max }(1 \mathrm{kHz})-T_{\max }(10 \mathrm{~Hz})\right]$ can be used to measure the degree of dispersion in relaxor materials [12]. These results are shown in Table 3 . There is no significant change seen in $\Delta T_{\max }$ for the two compositions measured. Further research into the dielectric relaxation in this system is ongoing.

Based on the above structural analysis and dielectric characterization, a partial phase diagram for the $(1-x) \mathrm{BT}-x \mathrm{BZN}$ solid solution is established, as shown in Figure 5. It 
delimits the tetragonal phase, a mixture of tetragonal and rhombohedral phases, and the cubic phase. In addition, it indicates the crossover from the ferroelectric phase to the relaxor state with increasing BZN concentration.

\subsection{Ferroelectric and Piezoelectric Properties}

Figure 6 shows the polarization $(\mathrm{P})$ - electric field (E) loops displayed for the selected ceramics of (1-x)BT- $x$ BZN solid solution, $\mathrm{x}=0.025$ and 0.05 , with $T_{\mathrm{C}}$ greater than room temperature. The ceramic of $x=0.025$ exhibits a slim $\mathrm{P}(\mathrm{E})$ loop (Inset of Fig. 6) because it experienced electric breakdown before a saturated loop could be obtained, indicating that the coercive field is greater than that of the breakdown field. The ceramic of $\mathrm{x}=0.05$, on the other hand, shows a "soft" $\mathrm{P}(\mathrm{E})$ loop with a remnant polarization Pr of about $5 \mu \mathrm{C} / \mathrm{cm}^{2}$ and a coercive field of $8 \mathrm{kV} / \mathrm{cm}$. This behaviour results from the fact that the transition temperature from the mixed rhombohedral and tetragonal ferroelectric phases to the cubic paraelectric phase is just above room temperature, as shown in the phase diagram of Fig. 5. It also reflects some precursor effect of the relaxor state which occurs at slightly higher BZN concentrations.

The piezoelectric measurement for the ceramics led to an average $d_{33}$ value of 22.7 $\mathrm{pC} / \mathrm{N}$ for the composition of $x=0.025$ when poled at $1.6 \mathrm{kV} / \mathrm{cm}$, and $16.2 \mathrm{pC} / \mathrm{N}$ for $x=$ 0.05 when poled at $10 \mathrm{kV} / \mathrm{cm}$, respectively. These values are significantly lower than that reported for pure $\mathrm{BaTiO}_{3}$ ceramics [17], which is mainly due to the very large coercive field in the $\mathrm{x}=0.025$ ceramic which prevents it from being poled effectively without breaking down, and the near-relaxor state in the $\mathrm{x}=0.05$ ceramic which diminishes its 
piezoelectric response. More work is underway to improve the piezoelectric response of this solid solution system.

\section{Conclusions}

The ceramics of a novel complex perovskite solid solution of $(1-x) \mathrm{BT}-x \mathrm{BZN}$ were synthesized by a solid state method and sintering process with a solubility limit of $x=$ 0.125. Compositions with $x \leq 0.025$ have a tetragonal symmetry, $x=0.05$ is a mixture of rhombohedral and tetragonal phases, and $x \geq 0.075$ have pseudo-cubic symmetry at room temperature. The substitution of BZN for BT is found to decrease the $T_{\mathrm{C}}$ and tetragonality of the material and eventually leads to a crossover from the ferroelectric state to relaxorlike behaviour. The $x=0.025$ and $x=0.05$ solid solutions have the $T_{\mathrm{C}}=98 \pm 3^{\circ} \mathrm{C}$ and $T_{\mathrm{C}}=$ $39 \pm 3^{\circ} \mathrm{C}$, respectively, and the $x \geq 0.075$ solid solutions exhibit frequency dispersion of the dielectric peaks, characteristic of relaxor behaviour. The increased diffuseness of the phase transition and the crossover to relaxor behaviour are attributed to increased chemical disorder arising from the aliovalent coupled substitutions of $\mathrm{Bi}^{3+}$ for $\mathrm{Ba}^{2+}$ and $\left(\mathrm{Zn}_{2 / 3} \mathrm{Nb}_{1 / 3}\right)^{3+}$ for $\mathrm{Ti}^{4+}$. A partial phase diagram of the $(1-x) \mathrm{BT}-x \mathrm{BZN}$ system is established based on these XRD analysis and dielectric measurements, which indicates the tetragonal phase, a mixture of tetragonal and rhombohedral phases, and the pseudo-cubic phase, as well as the crossover from the ferroelectric phase to the relaxor state with increasing BZN concentration. The decrease in $T_{\mathrm{C}}$ and tetragonality and the increase in phase symmetry at room-temperature with increasing BZN content are contrary to what is expected of the possible effects of BZN, as has been found in the PT-BZN solid solution where an increase in $T_{\mathrm{C}}$ and an enhanced tetragonality are observed with increased BZN concentration. These new results suggest that the BT-BZN solid solution possesses unique crystal chemistry and 
physics in terms of chemical disorder, lone electron pair effects and relaxation mechanism, which require further investigation.

\section{Acknowledgements}

The authors would like to thank Dr. N. Zhang for her help with XRD analysis. This work was supported by the United States Office of Naval Research (ONR, Grant No. N00014-12-1-1045) and the Natural Science and Engineering Research Council of Canada (NSERC). The work performed at XJTU was supported by the Natural Science Foundation of China (Grant Nos. 50728201 and 90923001), the International Science \& Technology Cooperation Program of China (Grant No. 2011DFA51880), the "111 Project" of China (Grant No. B14040). 


\section{References}

[1] S. Park and T.R. Shrout, Ultrahigh strain and piezoelectric behavior in relaxor based ferroelectric single crystals, J. Appl. Phys. 82 (1997) 1804-1811.

[2] S.E. Park and W. Hackenberger, High performance single crystal piezoelectrics: applications and issues, Curr. Opin. Solid State Mater. Sci. 6 (2002) 11-18.

[3] R.E. Eitel, C.A. Randall, T.R. Shrout and S.E. Park, Preparation and characterization of high temperature perovskite ferroelectrics in the solid-solution (1$x) \mathrm{BiScO}_{3}-x \mathrm{PbTiO}_{3}$, Jpn. J. Appl. Phys. 41 (2002) 2099-2104.

[4] R.E. Eitel, C.A. Randall, T.R. Shrout, P.W. Rehrig, W. Hackenberger, and S.E. Park, New high temperature morphotropic phase boundary piezoelectrics based on $\mathrm{Bi}(\mathrm{Me}) \mathrm{O}_{3}-\mathrm{PbTiO}_{3}$ ceramics, Jpn. J. Appl. Phys. 40 (2001) 5999-6002.

[5] I. Grinberg, M. Suchomel, W. Dmowski, S. Mason, H. Wu, P. Davies, and A. Rappe, Structure and polarization in the high $T_{\mathrm{C}}$ ferroelectric $\mathrm{Bi}(\mathrm{Zn}, \mathrm{Ti}) \mathrm{O}_{3}-\mathrm{PbTiO}_{3}$ solid solutions, Phys. Rev. Lett. 98 (2007) 107601.

[6] M.R. Suchomel, A.M. Fogg, M. Allix, H. Niu, J.B. Claridge and M.J. Rosseinsky, $\mathrm{Bi}_{2} \mathrm{ZnTiO}_{6}$ : A lead-free closed-shell polar perovskite with a calculated ionic polarization of $150 \mu \mathrm{C} \mathrm{cm}^{-2}$, Chem. Mater. 18 (2006) 4987-4989.

[7] M.R. Suchomel and P.K. Davies, Enhanced tetragonality in $(x) \mathrm{PbTiO}_{3^{-}}$ $(1-x) \mathrm{Bi}\left(\mathrm{Zn}_{1 / 2} \mathrm{Ti}_{1 / 2}\right) \mathrm{O}_{3}$ and related solid solution systems, Appl. Phys. Lett. 86 (2005) 262905.

[8] S. Nomura, K. Kaneta, J. Kuwata and K. Uchino, Phase transition in the $\mathrm{PbTiO}_{3}$ $\mathrm{A}\left(\mathrm{B}_{2 / 3} \mathrm{Nb}_{1 / 3}\right) \mathrm{O}_{3}(\mathrm{~A}=\mathrm{La}, \mathrm{Bi} ; \mathrm{B}=\mathrm{Zn}, \mathrm{Mg})$ solid solutions, Mater. Res. Bull. 17 (1982) 14711475. 
[9] A.A. Bokov and Z.-G. Ye, Recent progress in relaxor ferroelectrics with perovskite structure, J. Mater. Sci. 41 (2006) 31-52.

[10] H.N. Tailor, A.A. Bokov and Z.-G. Ye, Synthesis and dielectric characterization of a new relaxor solid solution of $(1-x) \mathrm{Pb}\left(\mathrm{Mg}_{1 / 3} \mathrm{Nb}_{2 / 3}\right) \mathrm{O}_{3}-x \mathrm{Bi}\left(\mathrm{Zn}_{1 / 2} \mathrm{Ti}_{1 / 2}\right) \mathrm{O}_{3}$, Ferroelectrics. 405 (2010) 67-75.

[11] N. Yasuda, H. Ohwa and S. Asano, Dielectric properties and phase transitions of $\mathrm{Ba}\left(\mathrm{Ti}_{1-x} \mathrm{Sn}_{x}\right) \mathrm{O}_{3}$, Jpn. J. of Appl. Phys. 35 (1996) 5099-5103.

[12] C. Lei, A.A. Bokov and Z.-G. Ye, Ferroelectric to relaxor crossover and dielectric phase diagram in the $\mathrm{BaTiO}_{3}-\mathrm{BaSnO}_{3}$ system, J. Appl. Phys. 101 (2007) 084105.

[13] N. Triamnak, R. Yimnirun, J. Pokorny and D. P. Cann, Relaxor characteristics of the phase transformation in $(1-x) \mathrm{BaTiO}_{3}-x \mathrm{Bi}\left(\mathrm{Zn}_{1 / 2} \mathrm{Ti}_{1 / 2}\right) \mathrm{O}_{3}$ perovskite ceramics, J. Am. Ceram. Soc. 96 (2013) 3176-3182.

[14] H. Ogihara, C.A. Randall and S. Trolier-McKinstry, Weakly coupled relaxor behavior of $\mathrm{BaTiO}_{3}-\mathrm{BiScO}_{3}$ ceramics, J. Am. Ceram. Soc. 92 (2009) 110-118.

[15] C.-C. Huang and D. P. Cann, Phase transitions and dielectric properties in $\mathrm{Bi}\left(\mathrm{Zn}_{1 / 2} \mathrm{Ti}_{1 / 2}\right) \mathrm{O}_{3}-\mathrm{BaTiO}_{3}$ perovskite solid solutions, J. Appl. Phys. 104 (2008) 024117.

[16] I. Fujii, K. Nakashima, N. Kumada and S. Wada, Structural, dielectric, and piezoelectric properties of $\mathrm{BaTiO}_{3}-\mathrm{Bi}\left(\mathrm{Ni}_{1 / 2} \mathrm{Ti}_{1 / 2}\right) \mathrm{O}_{3}$ ceramics, J. Ceram. Soc. Jpn. 120 (2012) 30-34.

[17] M. C. Cheung, H. L. W. Chan, and C. L. Choy, Study on barium titanate ceramics prepared by various methods, J. Mater. Sci. 36 (2001) 381-387. 


\section{Figure Captions}

Figure 1. X-ray diffraction patterns of the $(1-\mathrm{x}) \mathrm{BT}-\mathrm{xBZN}(\mathrm{x}=0.00-0.15)$ ceramics prepared at optimal sintering temperatures. Stars indicate impurity peaks in the $\mathrm{x}=0.15$ ceramics.

Figure 2. Rietveld refinement results of the XRD data for $x=0.025$ of $(1-x) B T-x B Z N$. The black line represents the experimental data, the red line represents the calculated fitting data, and the difference between these two sets of data is indicated by the grey line. The inset shows an enlarged view of the (200) splitting which is characteristic of the tetragonal phase.

Figure 3. Rietveld refinement results of the XRD data of the $(1-x) \mathrm{BT}-x \mathrm{BZN}$ solid solution with $x=0.05$ based on a model of coexisting tetragonal and rhombohedral phases. Inset (a) shows an enlarged view of the splitting of the (111) peak which is characteristic of the rhombohedral phase, and (b) shows the shoulder on the (200) peak, which is characteristic of the tetragonal phase.

Figure 4. Temperature dependence of the dielectric constant $\varepsilon$ ' measured at various frequencies upon cooling for various compositions of the $(1-x) \mathrm{BT}-x \mathrm{BZN}$ ceramics: (a) $x=$ 0.025 , (b) $x=0.05$, (c) $x=0.075$, and (d) $x=0.10$.

Figure 5. Partial phase diagram of the $(1-x) \mathrm{BT}-x \mathrm{BZN}$ solid solution system, indicating the tetragonal phase, a mixture of the tetragonal and rhombohedral phases, and the cubic phase, 
as well as a crossover from the ferroelectric phase to relaxor state with increasing BZN concentration. $T_{\max }$ was measured at $1 \mathrm{kHz}$.

Figure 6. Polarization versus electric field loops for the $(1-x) \mathrm{BT}-x \mathrm{BZN}$ ceramics $(x=0.025$ and 0.05$)$ with $T_{\mathrm{C}}$ above room temperature. 


\section{Tables}

Table 1. Calculated lattice parameters for the tetragonal phase and the tetragonality of the $(1-x)$ BT- $x$ BZN ceramics.

\begin{tabular}{cccc}
\hline Composition & $\boldsymbol{a}(\AA)$ & $\boldsymbol{c}(\AA)$ & $\boldsymbol{c} / \boldsymbol{a}$ \\
\hline$x=0$ & $3.9947 \pm 0.0006$ & $4.0386 \pm 0.0006$ & $1.0110 \pm 0.0002$ \\
$x=0.025$ & $4.0003 \pm 0.0002$ & $4.0290 \pm 0.0002$ & $1.00717 \pm 0.00007$ \\
\hline
\end{tabular}

Table 2. Calculated lattice parameters for the $(1-x) \mathrm{BT}-x \mathrm{BZN}$ ceramics of pseudo-cubic symmetry.

\begin{tabular}{cc}
\hline Composition & $\boldsymbol{a}(\mathbf{\AA})$ \\
\hline$x=0.075$ & $4.0117 \pm 0.0001$ \\
$x=0.10$ & $4.0143 \pm 0.0001$ \\
$x=0.125$ & $4.0156 \pm 0.0001$ \\
\hline
\end{tabular}

Table 3. The temperature difference $\Delta T_{\max }=\left[T_{\max }(1 \mathrm{kHz})-T_{\max }(10 \mathrm{~Hz})\right]$ of the dielectric peaks for the $x=0.075$ and 0.125 ceramics of the $(1-x) \mathrm{BT}-x \mathrm{BZN}$ solid solution.

\begin{tabular}{cc}
\hline Composition & $\Delta T_{\max }\left({ }^{\circ} \mathbf{C}\right)$ \\
\hline$x=0.075$ & $24 \pm 4$ \\
$x=0.10$ & $24 \pm 4$ \\
\hline
\end{tabular}




\section{Figures}

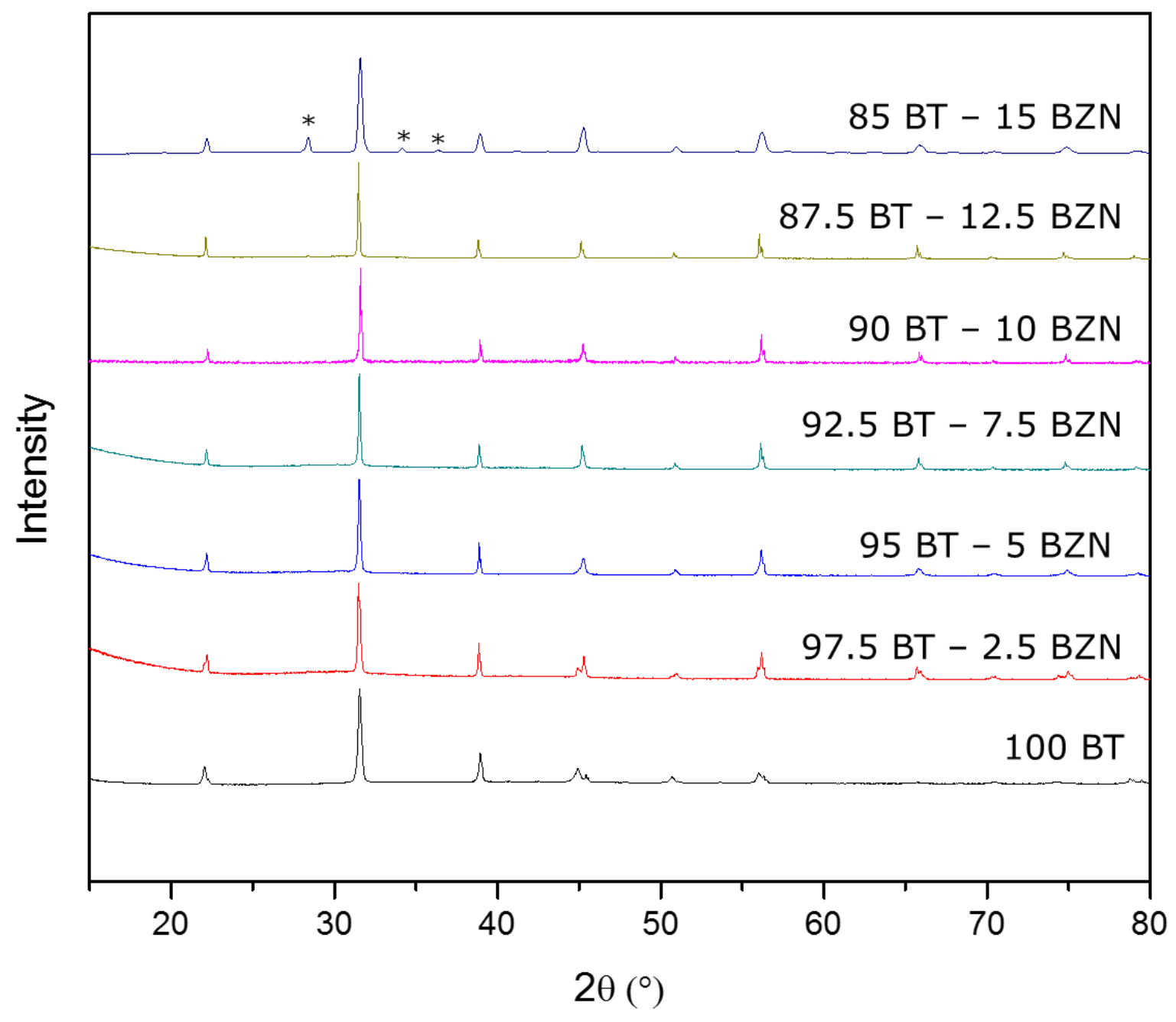

Figure 1 (Paterson et al.). 


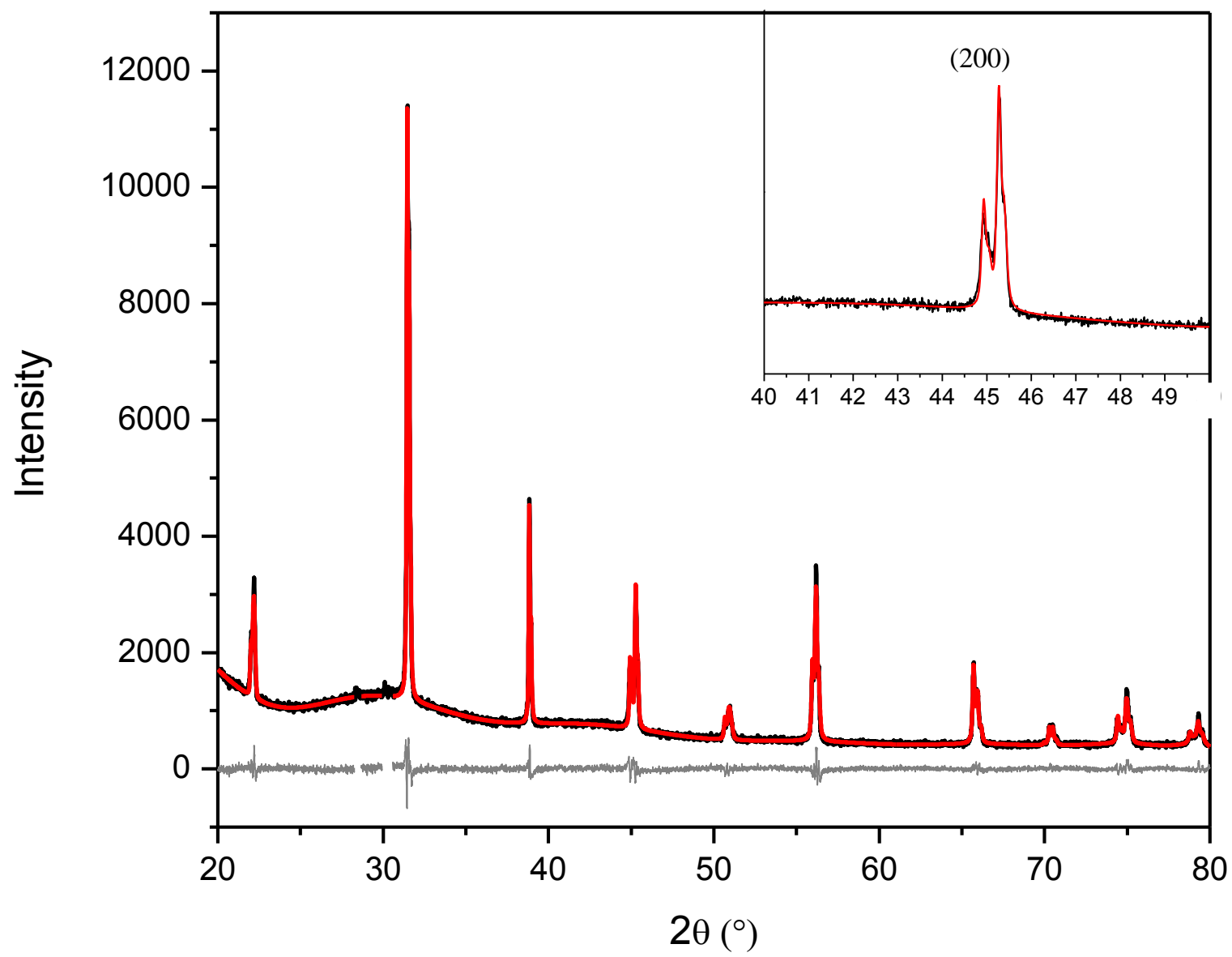

Figure 2 (Paterson et al.). 


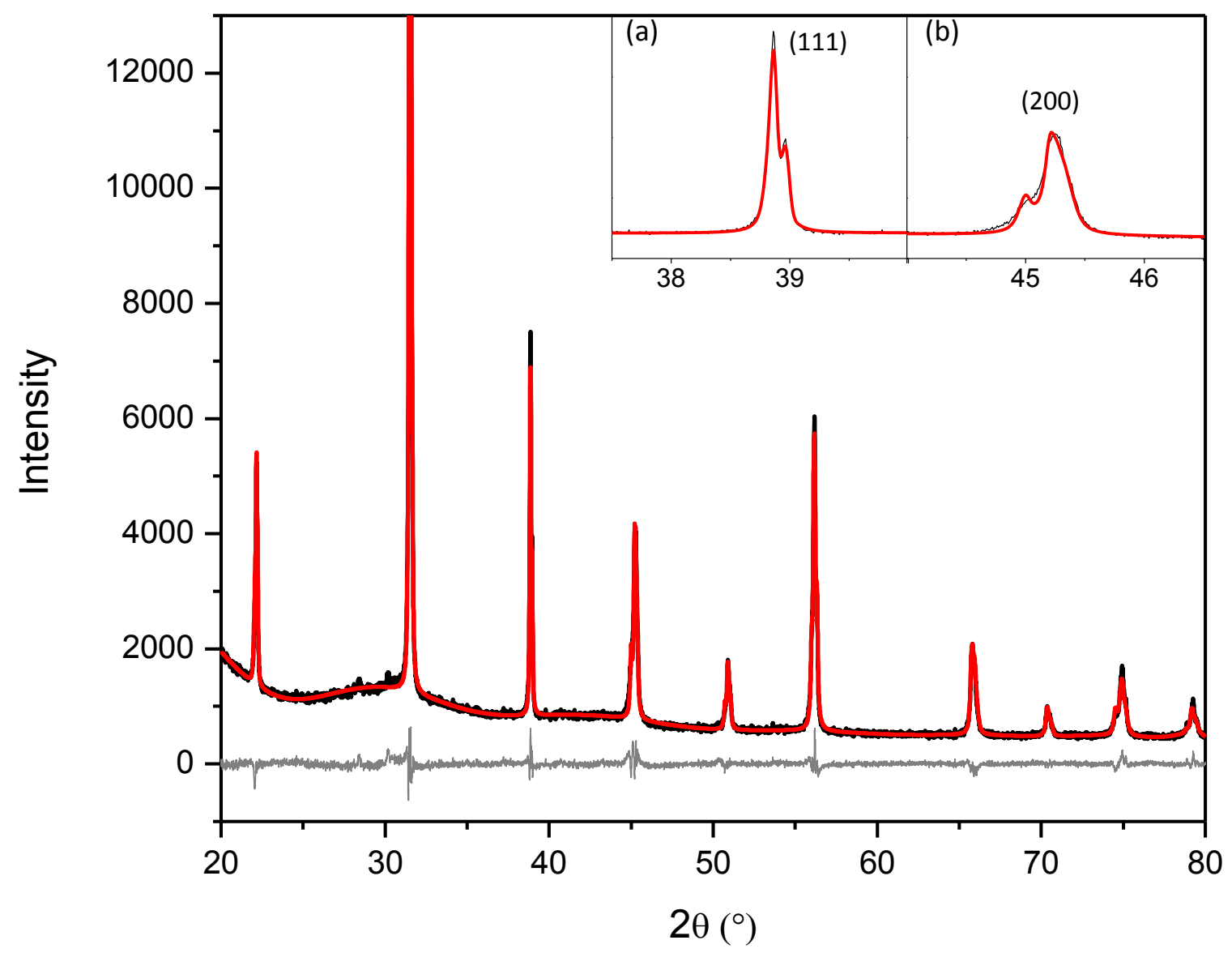

Figure 3 (Paterson et al.). 

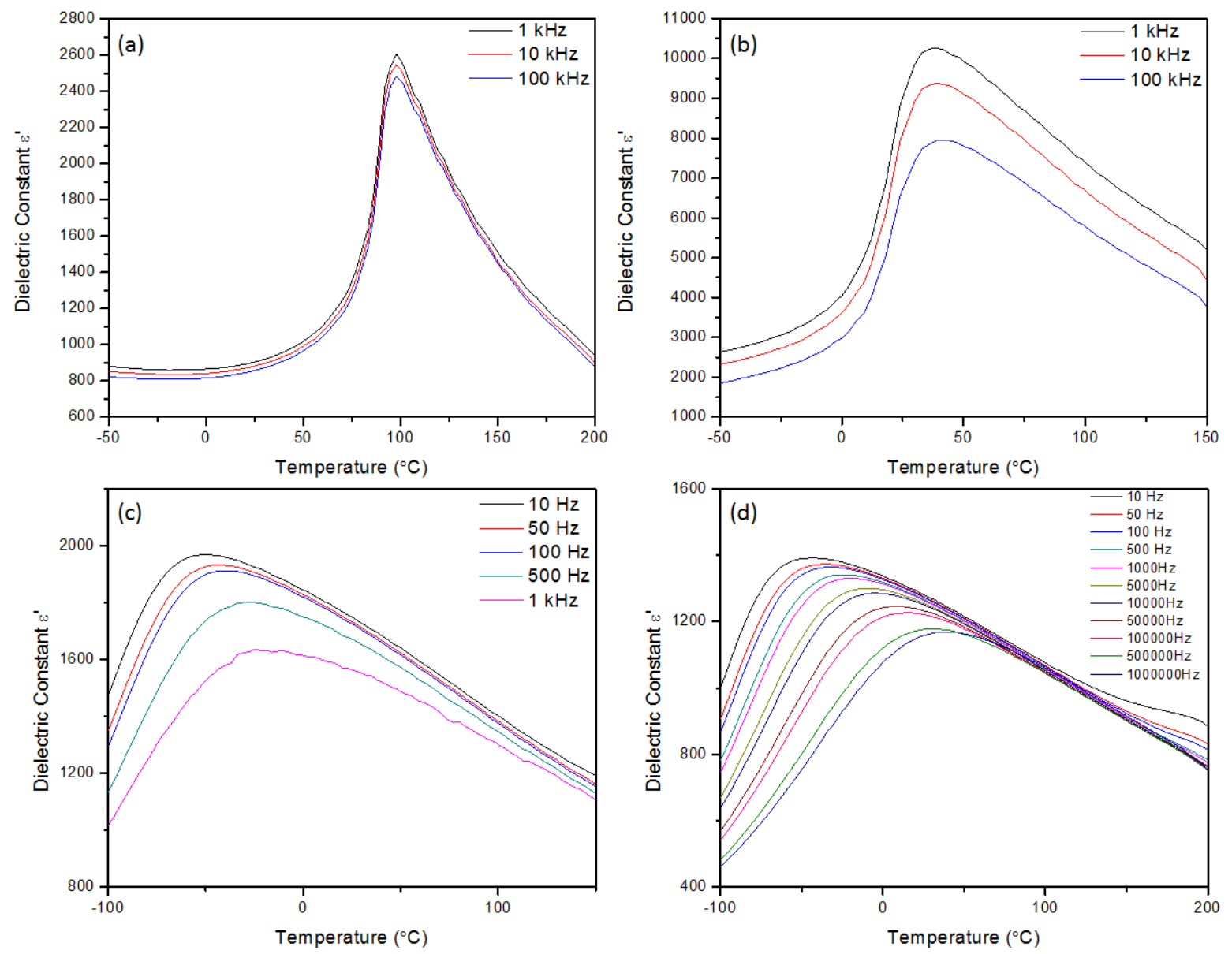

Figure 4 (a-d) (Paterson et al.). 


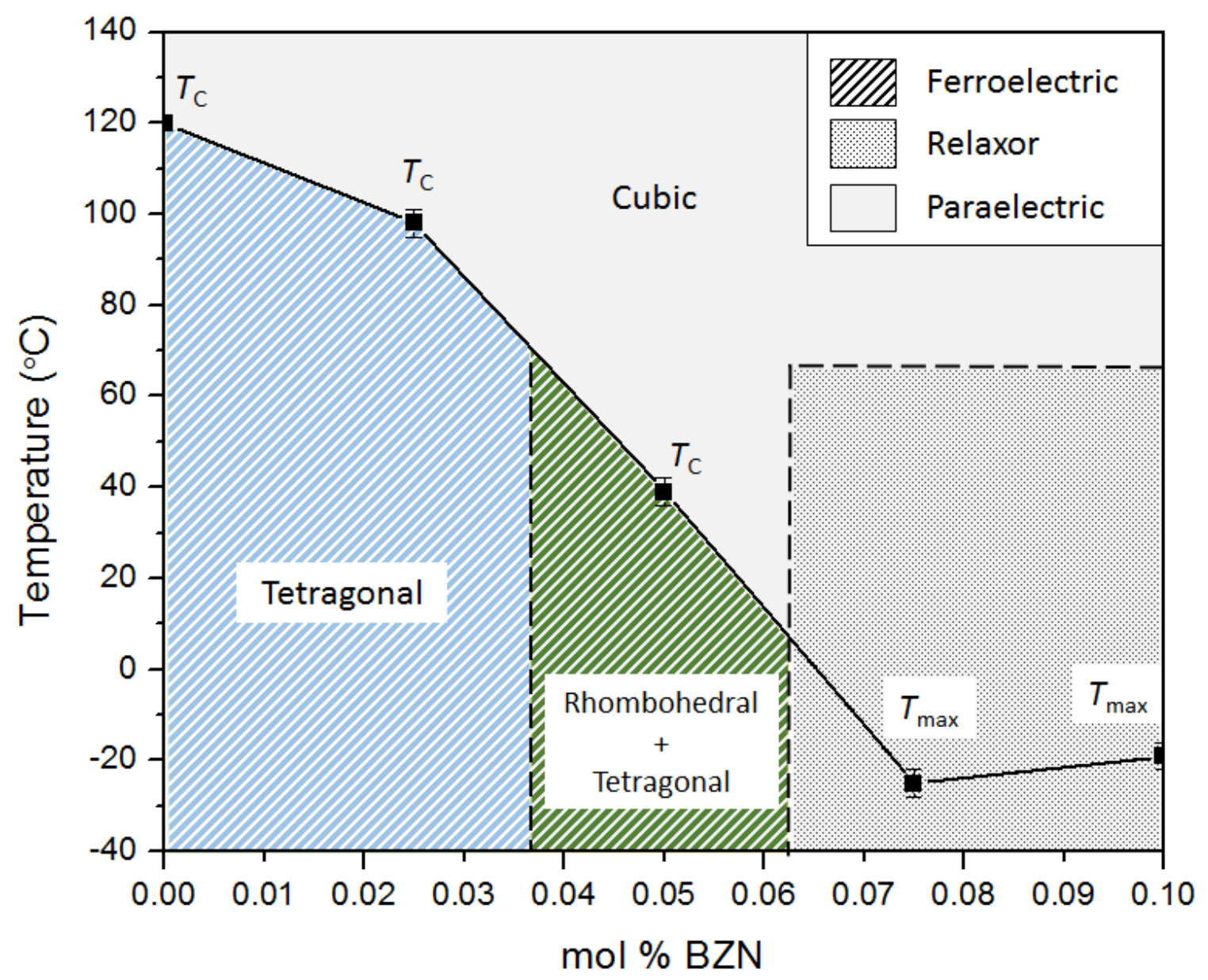

Figure 5 (Paterson et al.). 


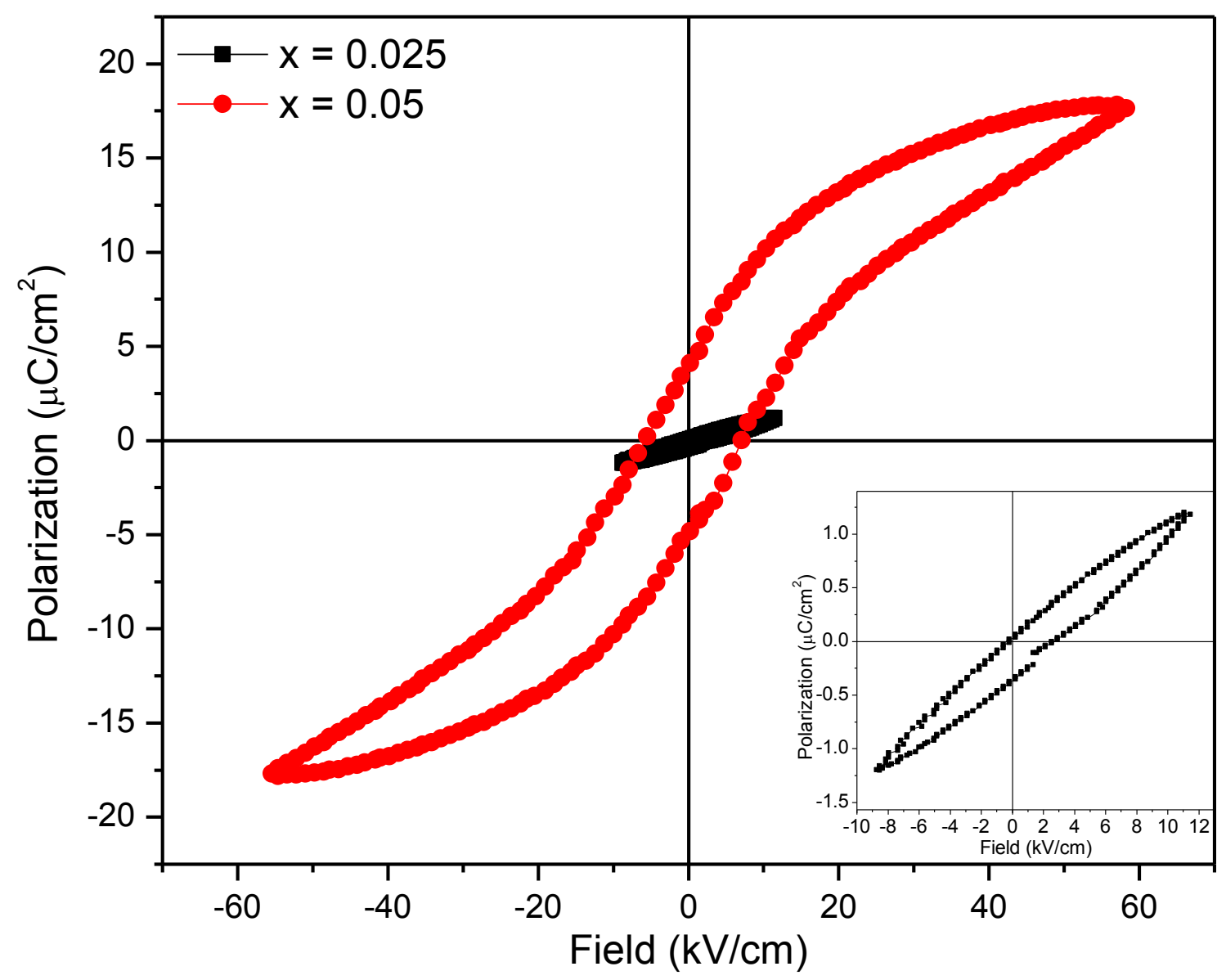

Figure 6 (Paterson et al.). 